\title{
Separatistische Bewegungen: Erklärungen und Lösungsansätze
}

Die turbulenten und sich überschlagenden Ereignisse in Katalonien haben das Thema Separatismus auf die Tagesordnung gebracht, de facto sind die jüngsten Ereignisse aber weniger ungewöhnlich als viele denken. Die schottische Unabhängigkeitsbewegung war 2014 kurz davor, sich erfolgreich von Großbritannien abzuspalten und die ETA kämpfte jahrzehntelang gewalttätig für ein unabhängiges Baskenland. Auch der Brexit kann am besten als eine separatistische Bewegung zur Abspaltung von einer größeren politischen Entität verstanden werden, wobei die Argumente der "Brexiteers“ denen anderer Unabhängigkeitsbewegungen verblüffend ähneln. Separatistische Bewegungen und Parteien existieren auf der ganzen Welt, aber während es einige zu Prominenz und politischem Erfolg gebracht haben, bleiben viele klein und erfolglos.

Die ökonomische Literatur über die optimale Größe eines Landes argumentiert, dass Regionen abwägen, welche Vor- und Nachteile es hat, einer gemeinsamen Nation anzugehören. Als Vorteile gelten größere Chancen für den gemeinsamen Handel und die effizientere Bereitstellung von öffentlichen Gütern. So ist ein gemeinsames Militär für alle deutschen Bundesländer zusammen wesentlich kostengünstiger als eine eigene Armee, da diverse Fixkosten aufgeteilt werden können. Als Hauptnachteil sieht die bestehende Literatur die teilweise Aufgabe politischer Selbstbestimmung, wenn die Präferenzen einer Region von den Präferenzen des restlichen Landes deutlich abweichen und sich die Bürger ungeliebten Mehrheitsbeschlüssen beugen müssen.

Ein wesentlicher Faktor, der in bisherigen Modellen vernachlässigt wird, ist die Ausstattung einer Region mit ökonomisch wertvollen Ressourcen. Der relative Reichtum einer Region (und damit das Besteuerungspotenzial) hängt von „natürlichen“ Ressourcen ab, aber auch von Ressourcen im weiteren Sinne, wie beispielsweise eine vorteilhafte geografische Lage, effizient arbeitende Institutionen, eine gute Infrastruktur oder vorhandenes Humankapital. In den meisten Ländern existieren Transfermechanismen, die auf diesen Ressourcen basierende Steuereinnahmen von reicheren zu ärmeren Regionen umverteilen. Diese Transfers sind oft der entscheidende Faktor für eine breite Unterstützung durch die Bevölkerung. Wichtig sind dabei nicht nur die umverteilten Summen, sondern auch die politischen Entscheidungsprozesse.

Eine politisch dominante Region hat z.B. wenig Anreize, sich vom Rest des Landes abzuspalten, da sie direkt über Umverteilungsmechanismen mitentscheiden kann. In Belgien war bis in die 1960er Jahre Wallonien, insbesondere dank der Verfügbarkeit natürlicher Ressourcen wie Kohle- und Erzvorkommen, die reichere und politisch dominante Region. Die größten Parteien zu dieser Zeit waren die Sozialisten und Christdemokraten, beide Parteien der Mitte. In den letzten Jahrzehnten verlor die Ressource „Kohle" stark an Wert, während der relativ höhere Bildungsstandard und der bedeutende Hafen in Antwerpen als Ressourcen für Flandern drastisch an Wert gewannen. Parallel dazu stieg die Unterstützung für Parteien wie die „Neu-Flämische Allianz", die Transferzahlungen und deren Ver(sch)wendung öffentlich anprangert; aktuell ist sie die stärkste Partei im belgischen Parlament.

Es stellt sich die Frage, ob regionaler Ressourcenreichtum den Erfolg separatistischer Parteien tatsächlich kausal miterklären kann, oder ob es sich um eine möglicherweise zufällige Korrelation handelt. Mithilfe eines natürlichen Experiments konnten wir diese Frage anhand von Wahlergebnissen in Großbritannien beantworten. Wir vergleichen dabei Wales mit Schottland: In beiden Regionen existieren seit dem frühen 20. Jahr-

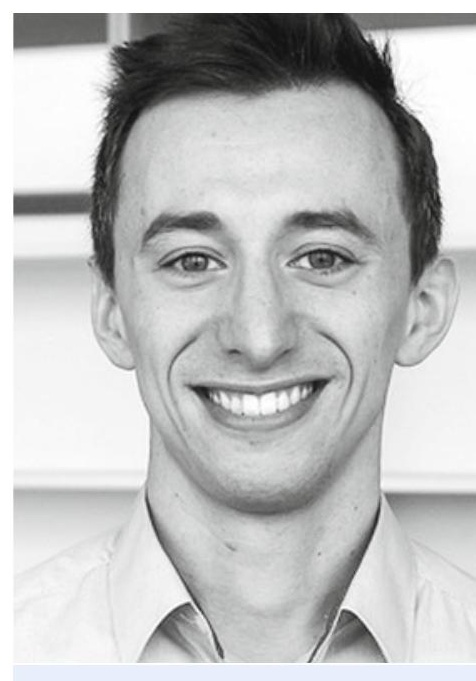

Dr. Kai Gehring ist Ökonom an der Universität Zürich. 
hundert mit Plaid Cymru und der Scottish National Party (SNP) separatistisch ausgerichtete Parteien. Im Gegensatz zu Wales erlebte Schottland aber seit 1970 durch die Ölfunde innerhalb seines hypothetischen Hoheitsgebiets einen drastischen Anstieg seines relativen Ressourcenreichtums. Die Wahlergebnisse beider Parteien verlaufen bis 1970 tatsächlich auf niedrigem Niveau quasi parallel, seit dem ersten großen Ölfund aber steigt der Stimmenanteil der SNP mit jedem Fund rasant an.

Kulturelle Faktoren allein sind also keine hinreichenden Erklärungen für den Erfolg separatistischer Parteien, in Einzelfällen nicht einmal notwendige Voraussetzung. Die durch die Lega Nord in Italien propagierte Republik „Padanien“ basiert beispielsweise nicht auf einem historischen Staat. Zweifellos ist eine starke regionale Identität hilfreich, aber in den allermeisten Fällen bedarf es zusätzlicher - ökonomischer - Argumente, um Wähler in großer Zahl zu begeistern. Zu dieser Erklärung passen auch unsere Schätzungen über die sinkende Unterstützung für die SNP mit fallenden Ölpreisen, höchstwahrscheinlich ein entscheidender Grund für das Scheitern des Referendums 2014. Auch katalanische Wähler werden die Reaktionen von Firmen, von denen Arbeitsplätze und Steuereinnahmen abhängen, in ihr weiteres Kalkül einbeziehen.

Die Analyse der Ursachen ist das eine, wichtig ist aber auch, welche Lösungen verfügbar sind. Die Unterdrückung einer Region durch einen zentralstaatlichen Akteur ist dabei sicher nicht hilfreich. Unsere Forschung zeigt am Beispiel Frankreichs, wie Unterdrückung und repressive Politik die gemeinsame Identität einer Region sogar verstärken kann. Betrachten wir die aktuellen Fälle, eint alle, dass sich der Ärger an als unfair empfundenen fiskalischen Transfersystemen entzündet. Daher ist die für die Zukunft der Nationalstaaten, wie auch der EU, entscheidende Frage: Lassen sich Transfersysteme konstruieren, die eine gewünschte Umverteilung oder Absicherung gegen asymmetrische Risiken ermöglichen, den Ärger der temporären oder dauerhaften Geberregionen aber minimieren? Dafür muss endlich ein konstruktiver Austausch zwischen politischen Ökonomen und Makroökonomen beginnen. Im Moment beharrt die eine Seite zu Recht, aber einseitig, auf den Nachteilen eines Transfersystems, während die andere einseitig, aber auch mit wichtigen Argumenten, die Vorteile betont.

Ich möchte hier folgende Hypothesen zur Diskussion stellen: Erstens, nicht nur die Rolle als Nettozahler an sich, sondern auch mangelnde Einflussmöglichkeiten auf die Verwendung der Gelder und mangelndes Vertrauen in diese Prozesse sorgen für Unzufriedenheit. Zweitens, die Finanzierung öffentlicher Projekte durch reine Transfers einer zentralen politischen Autorität bringt neben bekannten Nachteilen wie geringen Sparanreizen, mangelnder Transparenz und der unzureichenden Nutzung lokaler Informationen auch das aktuell sichtbare Problem der mangelnden Akzeptanz mit sich. Drittens, durch fremdes Geld finanzierte Projekte oder Dienstleistungen wie Autobahnen oder eine gebührenfreie Kinderbetreuung, die in Ausmaß oder Qualität über dem Niveau in den Geberregionen liegen, sorgen besonders für Unfrieden. Viertens, nicht alle Transferzahlungen sind gleich. Umverteilung über automatische Stabilisatoren, wie z.B. das Arbeitslosengeld oder das Rentensystem, führen eventuell zu weniger Unfrieden. Fünftens, eine hohe Steuerautonomie wie in der Schweiz, wo Gemeinden und Kantone einen großen Anteil ihrer Investitionsprojekte aus eigenen Mitteln finanzieren, kombiniert mit direkt-demokratischen Mitteln, ermöglicht die Einbeziehung der lokalen Bevölkerung und kann unerwünschte Ausgabenwünsche von Politikern begrenzen. Falls eine geografische Umverteilung angestrebt wird, muss die Allokation transparenten demokratischen Regeln folgen, die auch für die Bevölkerung

Kai Gehring

Universität Zürich

kai.gehring@pw.uzh.ch in den Geberregionen dauerhaft akzeptabel sind. Obwohl sie aus sehr heterogenen kulturellen Gruppen besteht, beweist die Schweiz, dass ein solches System nicht nur funktionieren, sondern auch stabil und sehr erfolgreich sein kann. 